CUBO A Mathematical Journal

Vol.14, No 01, (93-110). March 2012

\title{
New Aspects on Elementary Functions in the Context of Quaternionic Analysis
}

\author{
S. Georgiev \\ Department of Differential Equations, \\ University of Sofia, \\ Sofia, Bulgaria, \\ email: sgg2000bg@yahoo.com \\ J. MORAIS \\ Technical University of Mining, \\ Freiberg, Germany, \\ email: joao.pedro.morais@ua.pt \\ and \\ W. SPRÖsS \\ Technical University of Mining, \\ Freiberg, Germany, \\ email: sproessig@math.tu-freiberg.de
}

\begin{abstract}
The main objective of this article is to give a survey on elementary functions in the context of quaternionic analysis. We define some of their more common properties, which as in the real and complex cases, will be familiar to the reader. This leads to the consideration of quaternion-valued functions depending on a quaternion variable, that is, functions whose input and output are quaternions.
\end{abstract}




\section{RESUMEN}

El objetivo principal de este artículo es dar una visión general sobre funciones elementales en el contexto de análisis cuaterniónico. Definimos algunas de sus propiedades más comunes, que como en los casos reales y complejos, serán familiares para el lector. Esto lleva a la consideración de las funciones de valor-cuaterniónico dependiendo de una variable cuaterniónica, esto es, funciones las cuales de entrada y salida son cuaterniones.

Keywords and Phrases: Quaternionic analysis, elementary functions.

2010 AMS Mathematics Subject Classification: 30G35, 30A10.

\section{Introduction}

As is well known, quaternionic analysis generalizes the theory of holomorphic functions of one complex variable and also provides the foundations to refine the theory of harmonic functions in higher dimensions. Methods of quaternionic analysis in combination with other classical and modern analytical methods (such as harmonic analysis, variational methods, and finite difference methods) have been playing an increasingly active part in the treatment of problems, mainly in mathematical physics, which involve the treatment of elementary functions. Basic results were independently discovered and rediscovered by many people, among others: Scheffers (1893), Dixon (1904), Lanczos (1919), Moisil-Teodorescu (1931), Melijhzon (1948), Iftimie (1965), Hestenes (1968), Delanghe (1970), and Sudbery (1979), Brackx, Delanghe and Sommen (1982), Gürlebeck and W. Sprößig (1989). Meanwhile quaternionic analysis has became a well established branch in mathematics and greatly successful in many different directions. Navigation, computer vision, robotics, signal and image processing, or efficient description of classical mechanics and electrical engineering are examples of fields where quaternions are used nowadays. A survey within the scope of quaternionic analysis and its applications is given in [10, and references therein.

The organization of this paper is as follows. Section 2 begins with a review of some definitions and basic properties of quaternionic analysis. We proceed in Section 3 to study the quaternion exponential and logarithmic functions. Although quaternion multiplication is not commutative, many formal properties of the complex exponential and logarithmic functions can be generalized within this framework. In the remaining sections the quaternion trigonometric, hyperbolic, and their inverse functions are covered. A brief discussion on the notions of multiple-valued functions and branches is also presented. There is no attempt to cover everything related to elementary quaternion functions and review the historical development of quaternions. General information is contained in the books [6, 7]. 


\section{Basic properties and definitions for quaternions}

For all what follows we will work in $\mathbb{H}$, the skew field of real quaternions discovered by Hamilton in 1843. This means we can express each element $p \in \mathbb{H}$ uniquely in the form $p=p_{0}+p_{1} \mathbf{i}+p_{2} \mathbf{j}+p_{3} \mathbf{k}$, where $p_{i}(i=0,1,2,3)$ are ordinary numbers and the imaginary units $\mathbf{i}, \mathbf{j}$, and $\mathbf{k}$ stand for the elements of the basis of $\mathbb{H}$, subject to the multiplication rules

$$
\begin{aligned}
& \mathbf{i}^{2}=\mathbf{j}^{2}=\mathbf{k}^{2}=-1 \\
& \mathbf{i j}=\mathbf{k}=-\mathbf{j i}, \quad \mathbf{j k}=\mathbf{i}=-\mathbf{k j}, \quad \mathbf{k i}=\mathbf{j}=-\mathbf{i k}
\end{aligned}
$$

In this way the quaternionic algebra arises as a natural extension of the complex field $\mathbb{C}$. For, we identify $\mathbb{C}$ with the set of degenerate quaternions with zero coefficients of $\mathbf{j}$ and $\mathbf{k}$. We shall always assume the quaternion $p=0+0 \mathbf{i}+0 \mathbf{j}+0 \mathbf{k}:=0_{\mathbb{H}}$ to be the neutral element of addition, and $p=1+0 \mathbf{i}+0 \mathbf{j}+0 \mathbf{k}:=1_{\mathbb{H}}$ to be the multiplicative identity quaternion in the sequel. They are such that any quaternion added or multiplied by them remains unchanged. Further, we denote by

(i) $\operatorname{Sc}(p)=p_{0}$ the scalar part, and $\operatorname{Vec}(p)=\underline{p}=p_{1} \mathbf{i}+p_{2} \mathbf{j}+p_{3} \mathbf{k}$ the vector part of $p$,

(ii) $\bar{p}=p_{0}-\underline{p}$ the conjugate of $p$,

(iii) $|p|=\sqrt{p \bar{p}}=\sqrt{\bar{p} p}=\sqrt{p_{0}^{2}+p_{1}^{2}+p_{2}^{2}+p_{3}^{2}}$ the norm of $p$

(iv) $p^{-1}=\frac{\bar{p}}{|p|^{2}}, p \neq 0_{\mathbb{H}}$ the inverse of $p$

(v) $\operatorname{sgn}(p)=\frac{p}{|p|}$ the quaternion sign.

With the intention to obtain some insight for a forthcoming study of elementary quaternion functions, we now consider the classical polar form of a real quaternion with a modulus and an argument.

Theorem 2.1. Every real quaternion $\mathrm{p}$ with $\underline{\mathrm{p}} \neq(0,0,0)$ satisfies the trigonometric representation $p=|p|(\cos \theta+\operatorname{sgn}(\underline{p}) \sin \theta)$. Since $p_{0}^{2}+|\underline{p}|^{2}=|p|^{2}$ we find the relations:

$$
\cos \theta=\frac{p_{0}}{|p|}, \quad \text { and } \quad \sin \theta=\frac{|p|}{|p|} .
$$

The angle $\theta:=\arg (p)$ (with positive counterclockwise orientation) is called the quaternion argument of the quaternion $p$ and is only determined up to an integer multiple of $2 \pi$. For $p=0_{\mathbb{H}}$, $\arg (p)$ cannot be defined in any way that is meaningful. In practice, when $p \neq 0_{\mathbb{H}}$ we use $\tan \theta=\frac{|\underline{p}|}{p_{0}}$ to find $\theta$, where the quadrant in which $p_{0}$ and $|\underline{p}|$ lie must always be specified or be clearly understood. Although the symbol $\arg (p)$ actually represents a set of values, the $\operatorname{argument} \theta$ of a quaternion that lies in the interval $[0, \pi]$ is called the principal argument of $p$, and is represented 
by the symbol $\operatorname{Arg}(p)$.

We proceed with some fundamental definitions and notations which will be needed throughout the text.

Definition 2.1. The sequence $\left\{p_{n}\right\}_{n=1}^{\infty}$ of real quaternions $p_{n}$ is called convergent to the real quaternion $\mathrm{p}$ if for every $\epsilon>0$, there exists a natural number $\mathrm{N}$ such that for every $\mathrm{n}>\mathrm{N}$ we have $\left|\mathrm{p}_{\mathrm{n}}-\mathrm{p}\right|<\epsilon$. We will use the traditional notation: $\lim _{\mathrm{n} \longrightarrow \infty} \mathrm{p}_{\mathrm{n}}=\mathrm{p}$.

Definition 2.2. The sequence $\left\{\mathrm{p}_{\mathrm{n}}\right\}_{\mathfrak{n}=1}^{\infty}$ of real quaternions is called convergent to infinity if for every positive constant $\mathrm{M}$, there exists a natural number $\mathrm{N}$ such that for every $\mathrm{n}>\mathrm{N}$ we have $\left|p_{n}\right| \geq M$. For this one uses the notation: $\lim _{n \rightarrow \infty} p_{n}=\infty$.

Definition 2.3. The sequence $\left\{p_{n}=p_{0, n}+p_{1, n} i+p_{2, n} j+p_{3, n} k_{n=1}^{\infty}, p_{m, n} \in \mathbb{R}(m=0,1,2,3)\right.$ is called convergent to $-\infty$ and we write $\lim _{n \longrightarrow \infty} p_{n}=-\infty$ if for every constant $A \in \mathbb{R}$, there exists $\mathrm{N}=\mathrm{N}(\mathrm{A})$ such that for every $\mathrm{n}>\mathrm{N}$ we have $\mathrm{p}_{\mathrm{m}, \mathrm{n}}<\mathrm{A}, \mathrm{m}=0,1,2,3$.

Let us go on to the consideration of quaternion-valued functions. A quaternion function of a quaternion variable $p$, or, briefly, an $\mathbb{H}$-valued function is a function whose input and output are quaternions. It is a mapping $f: \mathbb{H} \longrightarrow \mathbb{H}$ such that $f(p)=[f(p)]_{0}+[f(p)]_{1} \mathbf{i}+[f(p)]_{2} \mathbf{j}+[f(p)]_{3} \mathbf{k}$, where the coordinate-functions $[f]_{\mathfrak{i}}(i=0,1,2,3)$ are real-valued functions defined in $\mathbb{H}$. As in the case with complex functions we have the standard operations on quaternion functions. In particular, given two functions $f(p)$ and $g(p)$, we define addition (subtraction), $f(p) \pm g(p)$, conjugation $\bar{f}(p)=\overline{f(p)}$, multiplication $f(p) g(p)$, and quotient $\frac{f(p)}{g(p)}$ provided with $g(p) \neq 0_{\mathbb{H}}$. We shall always write $\frac{f(p)}{g(p)}$ to mean $f(p) g^{-1}(p)$ in the sequel. Note that this is generally different from $g^{-1}(p) f(p)$ since quaternion multiplication is not commutative. Implicit in the previous arithmetic operations are simple but very important facts: $[f]_{0}=\frac{1}{2}(f+\bar{f})$ and $\underline{f}=\operatorname{Vec}(f)=\frac{1}{2}(f-\bar{f})$. On

occasion the modulus of a quaternion function $f$ is defined by $|f|=\sqrt{f \bar{f}}=\sqrt{\overline{f f}}=\sqrt{\sum_{i=0}^{3}[f]_{i}^{2}}$, and coincides with its corresponding Euclidean norm as a vector in $\mathbb{R}^{4}$.

\section{Exponents and Logarithms revisited}

In this section we revisit the quaternionic analogues of the complex exponential and logarithmic functions. It turns out that exponential and logarithmic quaternion functions can be defined because quaternions have a division algebra. The principal value of the quaternion logarithm will be defined to be a single-valued function whose argument lies in the interval $[0, \pi]$. This principal value quaternion function will be shown to be an inverse of the quaternion exponential function defined on a suitably restricted domain of the quaternion space.

We therefore start our discussion with the following definition. 
Definition 3.1. The function $e^{p}$ defined by $e^{p}:=\sum_{k=0}^{\infty} \frac{p^{k}}{k !}$ is called the quaternion natural exponential function.

In the case that $p$ is a complex number, the definition of $e^{\mathfrak{p}}$ is naturally extended to comply with the usual exponential function of complex numbers. Analogous to the complex case one may derive a closed-form representation for the quaternion exponential function.

Theorem 3.1. For the quaternion natural exponential function the following representation holds:

$$
e^{\mathfrak{p}}=e^{\mathfrak{p}_{0}}(\cos |\underline{p}|+\operatorname{sgn}(\underline{p}) \sin |\underline{p}|) .
$$

Corollary 3.1. Let $\left\{p_{n}\right\}_{n=1}^{\infty}$ be a sequence of elements of $\mathbb{H}$ such that $\lim _{n \rightarrow \infty} p_{n}=p$. The usual limit representation works:

$$
\lim _{n \rightarrow \infty}\left(1+\frac{p_{n}}{n}\right)^{n}=e^{p}
$$

We now record some useful properties of the quaternion exponential function.

Corollary 3.2. (see [6, 13, 7]) The quaternion natural exponential function satisfies the following properties:

(1) $e^{\mathfrak{p}} \neq 0_{\mathbb{H}}$, for all $p \in \mathbb{H}$,

(2) $e^{-\mathfrak{p}} e^{p}=1_{\mathbb{H}}, \quad e^{\operatorname{sgn}(\underline{p}) \pi}=-1_{\mathbb{H}}$,

(3) $\left(e^{\mathfrak{p}}\right)^{\mathfrak{n}}=e^{\mathfrak{n} \mathfrak{p}}$ for $\mathfrak{n}=0, \pm 1, \pm 2, \ldots$ (de Moivre's formula),

(4) $e^{\mathfrak{p}_{1}} e^{\mathfrak{p}_{2}} \neq e^{\mathfrak{p}_{1}+p_{2}}$ in general, unless $\mathrm{p}_{1}$ and $\mathrm{p}_{2}$ commute,

(5) $\left|e^{\mathfrak{p}}\right|=e^{\mathrm{Sc}(\mathfrak{p})}$

(6) $\overline{e^{p}}=e^{\bar{p}}$.

In what follows we introduce the quaternion natural logarithm function $\ln (p)$, which is motivated as defining the "inverse" of the quaternion natural exponential function $e^{\mathfrak{p}}$. More precisely, we define $\ln (p)$ to be any quaternion number such that $e^{\ln (p)}=p$ and $\ln \left(e^{p}\right)=p$. This is too much to hope for. We shall discuss this matter in further detail in the remainder of the section.

Definition 3.2. The multiple-valued quaternion natural logarithm function $\ln (\mathrm{p})$ is defined by

$$
\ln (p)=\log _{e}|p|+\operatorname{sgn}(\underline{p}) \arg (p) .
$$


Here $\log _{e}|p|$ is the usual real natural logarithm of the positive number $|p|$. By switching to polar form in (3.1), we obtain the following alternative description of the quaternion logarithm:

$$
\begin{aligned}
& \ln (\mathbf{p})= \begin{cases}\log _{e}|p|+\operatorname{sgn}(\underline{p})\left(\arccos \frac{p_{0}}{|p|}+2 \pi n\right) & ,|\underline{p}| \neq 0 \\
\log _{e}\left|p_{0}\right| & ,|\underline{p}|=0\end{cases} \\
& = \begin{cases}\log _{e}|p|+\operatorname{sgn}(\underline{p})\left(\arctan \frac{|\underline{p}|}{p_{0}}+2 \pi n\right) & , p_{0}>0 \\
\log _{e}|\underline{p}|+\operatorname{sgn}(\underline{p})\left(\frac{\pi}{2}+2 \pi n\right) & , p_{0}=0\end{cases}
\end{aligned}
$$

where $\mathrm{n}=0, \pm 1, \pm 2, \ldots$

Theorem 3.2. Let $\left\{\mathrm{p}_{\mathrm{n}}\right\}_{\mathrm{n}=1}^{\infty}$ be a sequence of elements of $\mathbb{H}$ and $\mathrm{n} \in \mathbb{N}$. The following statements are valid:

(1) If $\lim _{n \longrightarrow \infty} p_{n}=\infty$ then $\lim _{n \longrightarrow \infty} e^{p_{n}}=\infty$,

(2) If $\lim _{n \longrightarrow \infty} p_{n}=\infty$ then $\lim _{n \longrightarrow \infty} \ln \left(p_{n}\right)=\infty$ and $\lim _{n \longrightarrow \infty} \frac{\ln \left(p_{n}\right)}{p_{n}}=0$,

(3) If $\lim _{n \longrightarrow \infty} p_{n}=0$ then $\lim _{n \longrightarrow \infty} \ln \left(p_{n}\right)=-\infty$,

(4) If $\lim _{\mathrm{n} \longrightarrow \infty} p_{\mathrm{n}}=\mathrm{p}$ then $\lim _{\mathrm{n} \longrightarrow \infty} \ln \left(\mathrm{p}_{\mathrm{n}}\right)=\ln (\mathrm{p})$.

Proof. These statements follow from Definitions 3.1 and 3.2 .

If we wish to define single-valued branches of $\ln (p)$, it would be more satisfactory to restrict $\arg (p)$ to its principal value $\operatorname{Arg}(p)$. This yields to the following definition.

Definition 3.3. The principal value of the quaternion logarithm is denoted by the symbol $\operatorname{Ln}(\mathrm{p})$, and is defined as

$$
\operatorname{Ln}(p)=\log _{e}|p|+\operatorname{sgn}(\underline{p}) \operatorname{Arg}(p)
$$

At this stage we return to the principle mentioned at the beginning of the section. Since $\operatorname{Ln}(p)$ is one of the values of the quaternion logarithm $\ln (p)$, it follows from 3.2 that: $e^{\operatorname{Ln}(p)}=p$ and $\operatorname{Ln}\left(e^{p}\right)=p$ for all nonzero quaternion $p$ defined on the so-called fundamental region $p_{0}>0$ and $|\underline{p}|<\pi$. This suggests that the quaternion function $\operatorname{Ln}(p)$ plays the role of an inverse function of the exponential quaternion function $e^{\mathfrak{p}}$. To justify this claim, observe that for the quaternion $p=\pi k$, which is clearly not in the fundamental region, we have: $e^{\operatorname{Ln}(\pi k)}=e^{\log _{e}|\pi|} k=\pi k$, but $\operatorname{Ln}\left(e^{\pi k}\right)=\operatorname{Ln}(-1)=0$.

Corollary 3.3. The principal value of the quaternion logarithm function retains the following properties: 
(1) $e^{\operatorname{Ln}(p)}=p$ and $\operatorname{Ln}\left(e^{\mathfrak{p}}\right)=p$, for all nonzero quaternion $p$ defined on the fundamental region $p_{0}>0$ and $|p|<\pi$,

(2) $\operatorname{Ln}(1)=0, \operatorname{Ln}(\mathfrak{i})=\frac{\pi}{2} \mathfrak{i}, \operatorname{Ln}(\mathfrak{j})=\frac{\pi}{2} \mathfrak{j}$, and $\operatorname{Ln}(\mathrm{k})=\frac{\pi}{2} \mathrm{k}$,

(3) $\operatorname{Ln}\left(\mathrm{p}_{1} \mathrm{p}_{2}\right) \neq \operatorname{Ln}\left(\mathrm{p}_{1}\right)+\operatorname{Ln}\left(\mathrm{p}_{2}\right)$ in general, unless $\mathrm{p}_{1}$ and $\mathrm{p}_{2}$ commute,

(4) $\operatorname{Ln}\left(\mathrm{p}^{\mathfrak{n}}\right)=\mathrm{nLn}(\mathrm{p})$, for $\mathrm{n}=0, \pm 1, \pm 2, \ldots$ (de Moivre's formula).

Proof. The proofs follow immediately from Definition 3.3 .

In this connection it is of interest to note:

Theorem 3.3. Let $\mathrm{p}$ be a real quaternion such that $|\mathrm{p}| \geq 1$. The following inequalities are valid:

(1) $|\operatorname{Ln}(p)| \leq|p|-1+\pi$,

(2) $|\operatorname{Ln}(\mathfrak{p})| \leq \frac{2|\mathfrak{p}|^{3}-9|\mathbf{p}|^{2}+18|\mathfrak{p}|-11}{6}+\pi$,

(3) $|\operatorname{Ln}(p)| \leq \sum_{k=1}^{2 n-1} \frac{(|p|-1)^{k}}{k}+\pi, \quad n \in \mathbb{N}$

Proof. For the proof of Statement 1. a first straightforward computation shows that

$$
|\operatorname{Ln}(p)| \leq\left|\log _{e}(1+|p|-1)\right|+|\operatorname{sgn}(\underline{p}) \operatorname{Arg}(p)| \leq|p|-1+\pi .
$$

The last step follows from the standard inequality $\ln (1+x) \leq x$ for every $x \geq 0$. In a similar manner, to prove Statement 2. we shall use the inequality $\ln (1+x) \leq x-\frac{x^{2}}{2}+\frac{x^{3}}{3}$ for every $x \geq 0$. Equally clear is the following

$$
|\operatorname{Ln}(p)| \leq(|p|-1)-\frac{(|p|-1)^{2}}{2}+\frac{(|p|-1)^{3}}{3}+\pi=\frac{2|p|^{3}-9|p|^{2}+18|p|-11}{6}+\pi .
$$

Lastly, the proof of Statement 3. is a consequence of the classical inequality $\ln (1+x) \leq \sum_{k=1}^{2 n-1}(-1)^{k+1} \frac{x^{k}}{k}$ for every $x \geq 0$.

To supplement our investigations, we shall now introduce a general quaternion power function.

Definition 3.4. If $\mathrm{q}$ is a real quaternion and $\mathrm{p} \neq 0_{\mathbb{H}}$, then the quaternion power function $\mathrm{p}^{\mathrm{q}}$ is defined to be:

$$
p^{q}=e^{\ln (p) q} .
$$


One fact that should be stressed here is that depending on $\mathrm{q}$ the quaternion power function will have either one, finitely many or infinitely many values: If $q=n$ is an integer then $p^{n}$ assumes only one value. If $q=\frac{a}{b}$ is a rational number, where $a$ and $b$ are common factors, then $p^{\frac{a}{b}}=|p|^{\frac{a}{b}} e^{\operatorname{sgn}(\underline{p}) \arg (p) \frac{a}{b}}$ may have a finite number of values. If $q$ is a nonzero real quaternion, then $\mathrm{p}^{\mathrm{q}}$ may always have an infinite number of values.

Corollary 3.4. Quaternion powers satisfy the following properties:

(1) $\left(p^{q}\right)^{n}=p^{n q}$ for $n=0, \pm 1, \pm 2, \ldots$,

(2) $p^{q_{1}} p^{q_{2}} \neq p^{q_{1}+q_{2}}$ in general, unless $\ln (p) q_{1}$ and $\ln (p) q_{2}$ commute.

Proof. The proof follows immediately from Definition 3.4 .

We shall conclude our considerations with some of the more common properties of the abovementioned elementary quaternion functions, which as in the real and complex cases, will be familiar to the reader.

Theorem 3.4. Let $\mathrm{p}$ be a real quaternion, and $\mathrm{n} \in \mathbb{N}$. Then

$$
\lim _{n \rightarrow \infty} n(\sqrt[n]{p}-1)=\log _{e}|p|
$$

Proof. Using previous definitions a first straightforward computation shows that

$$
\begin{aligned}
\sqrt[n]{p} & =e^{\frac{1}{n} \log _{e}|p|}\left[\cos \left|\frac{1}{n} \operatorname{sgn}(\underline{p}) \arg (p)\right|+\operatorname{sgn}\left(\frac{1}{n} \operatorname{sgn}(\underline{p}) \arg (p)\right) \sin \left|\frac{1}{n} \operatorname{sgn}(\underline{p}) \arg (p)\right|\right] \\
& =e^{\frac{1}{n} \log _{e}|p|}\left[\cos \left|\frac{\arg (p)}{n}\right|+\operatorname{sgn}(\underline{p}) \sin \left|\frac{\arg (p)}{n}\right|\right] .
\end{aligned}
$$

This yields

$$
\begin{aligned}
\lim _{n \rightarrow \infty} n(\sqrt[n]{p}-1) & =\lim _{n \rightarrow \infty} \frac{e^{\frac{1}{n} \log _{e}|p|\left[\cos \left|\frac{\arg (p)}{n}\right|+\operatorname{sgn}(\underline{p}) \sin \left|\frac{\arg (p)}{n}\right|\right]-1}}{\frac{1}{n}} \\
& =\log _{e}|p| .
\end{aligned}
$$

Theorem 3.5. Let $\mathrm{p}$ be a real quaternion, and $\mathrm{n} \in \mathbb{N}$. Then

$$
\lim _{n \rightarrow \infty}\left(\frac{1+\sqrt[n]{p}}{2}\right)^{n}=\sqrt{|p|}
$$


Proof. Obviously, one has

$$
\frac{1+\sqrt[n]{p}}{2}=1+\frac{n(\sqrt[n]{p}-1)}{2 n}
$$

Hence in accordance with the previous result, it follows

$$
\lim _{n \rightarrow \infty}\left(\frac{1+\sqrt[n]{p}}{2}\right)^{n}=\lim _{n \longrightarrow \infty}\left(1+\frac{n(\sqrt[n]{p}-1)}{2 n}\right)^{n}=e^{\frac{\log _{e}|p|}{2}}=\sqrt{|p|} .
$$

Having treated these special cases, we may now pass to a somewhat more general case in which a finite sequence of quaternions takes a part in the definitions of the above-mentioned quaternion elementary functions.

Theorem 3.6. Let $\mathrm{k} \in \mathbb{N}$, and $\left\{\mathrm{p}_{v}\right\}_{v=1}^{\mathrm{k}}$ be a sequence of elements of $\mathbb{H}$. Then

$$
\lim _{n \longrightarrow \infty}\left(\frac{1}{k} \sum_{v=1}^{k} \sqrt[n]{p_{v}}\right)^{n}=\sqrt[k]{\prod_{v=1}^{k}\left|p_{v}\right|}
$$

Proof. We have clearly

$$
\frac{1}{k} \sum_{v=1}^{k} \sqrt[n]{p_{v}}=1+\frac{1}{k n}\left[n \sum_{v=1}^{k}\left(\sqrt[n]{p_{v}}-1\right)\right]
$$

and, as the above discussion shows, it follows

$$
\lim _{n \rightarrow \infty} \frac{1}{k}\left[n \sum_{v=1}^{k}\left(\sqrt[n]{p_{v}}-1\right)\right]=\frac{1}{k} \sum_{v=1}^{k} \log _{e}\left|p_{v}\right|=\log _{e} \sqrt[k]{\prod_{v=1}^{k}\left|p_{v}\right|}
$$

With these calculations at hand, we set

$$
\lim _{n \longrightarrow \infty}\left(\frac{1}{k} \sum_{v=1}^{k} \sqrt[n]{p_{v}}\right)^{n}=e^{\log _{e} \sqrt[k]{\prod_{v=1}^{k}\left|p_{v}\right|}}=\sqrt[k]{\prod_{v=1}^{k}\left|p_{v}\right|}
$$

As concerns the definition of quaternion power function, we can formulate the next theorems.

Theorem 3.7. Let $\mathrm{p}_{1}$ and $\mathrm{p}_{2}$ be two real quaternions, and $\mathrm{n} \in \mathbb{N}$. Then

$$
\lim _{n \rightarrow \infty} \frac{\left(\sqrt[n]{p_{1}}+\sqrt[n]{p_{2}}\right)^{n}}{2^{n}}=1 .
$$


Proof. From Definition 3.4 , a direct computation shows that

$$
\begin{aligned}
& \sqrt[n^{2}]{p_{1}}=e^{\frac{1}{n^{2}} \log _{e}\left|p_{1}\right|}\left[\cos \left|\frac{\arg \left(p_{1}\right)}{n^{2}}\right|+\operatorname{sgn}\left(\underline{p_{1}}\right) \sin \left|\frac{\arg \left(p_{1}\right)}{n^{2}}\right|\right], \\
& \sqrt[n]{p_{2}}=e^{\frac{1}{n^{3}} \log _{e}\left|p_{2}\right|}\left[\cos \left|\frac{\arg \left(p_{2}\right)}{n^{3}}\right|+\operatorname{sgn}\left(\underline{p_{2}}\right) \sin \left|\frac{\arg \left(p_{2}\right)}{n^{3}}\right|\right],
\end{aligned}
$$

and therefore, it follows

$$
\lim _{n \longrightarrow \infty} n\left(\sqrt[n^{2}]{p_{1}}-1\right)=0, \quad \text { and } \quad \lim _{n \longrightarrow \infty} n\left(\sqrt[n]{p_{2}}-1\right)=0 .
$$

Accordingly, this leads to

$$
\lim _{n \rightarrow \infty} \frac{\left(\sqrt[n^{2}]{p_{1}}+\sqrt[n]{p_{2}}\right)^{n}}{2^{n}}=\lim _{n \rightarrow \infty}\left[1+\frac{n\left(\sqrt[n]{p_{1}}-1\right)+n\left(\sqrt[n]{p_{2}}-1\right)}{2 n}\right]^{n}=1 .
$$

Theorem 3.8. Let $\left\{p_{n}\right\}_{n=1}^{\infty}$ and $\left\{q_{n}\right\}_{n=1}^{\infty}$ be two sequences of elements of $\mathbb{H}(n \in \mathbb{N})$ such that $\lim _{n \longrightarrow \infty} p_{n}=p$, and $\lim _{n \longrightarrow \infty} q_{n}=q$. Then

$$
\lim _{n \rightarrow \infty} \frac{1}{2^{n}}\left(\sqrt[n]{p_{n}}+\sqrt[n]{q_{n}}\right)^{n}=\sqrt{|p \| q|}
$$

Proof. The proof follows immediately from Theorem 3.6 .

Theorem 3.9. Let $\left\{p_{n}^{k}\right\}_{n=1}^{\infty}$ be a sequence of elements of $\mathbb{H}(n, k \in \mathbb{N})$ such that $\lim _{n \rightarrow \infty} p_{n}^{k}=p^{k}$. Then

$$
\lim _{n \rightarrow \infty}\left(\frac{1}{k} \sum_{v=1}^{k} \sqrt[n]{p_{n}^{v}}\right)=\sqrt[k]{\prod_{v=1}^{k}\left|p^{v}\right|}
$$

Proof. The proof is a consequence of Theorem 3.6 .

\section{Trigonometry revisited}

In this section we define quaternion trigonometric functions. Analogously to the quaternion functions $e^{\mathfrak{p}}$ and $\ln (p)$, these functions will agree with their counterparts for complex input. In addition, we will notice that the quaternion trigonometric functions satisfy various of the same identities as the real and complex trigonometric functions.

With the help of the exponential function, quaternionic analogues of the trigonometric functions can be introduced. 
Definition 4.1. The functions $\sin (\mathrm{p})$ and $\cos (\mathrm{p})$ defined respectively by

$$
\begin{aligned}
& \sin (\mathfrak{p})= \begin{cases}-\frac{1}{2} \operatorname{sgn}(\underline{p})\left(e^{p \operatorname{sgn}(\underline{p})}-e^{-p \operatorname{sgn}(\underline{p})}\right) & ,|\underline{p}| \neq 0 \\
\sin \left(p_{0}\right) & ,|\underline{p}|=0\end{cases} \\
& \cos (p)= \begin{cases}\frac{1}{2}\left(e^{p \operatorname{sgn}(\underline{p})}+e^{-p \operatorname{sgn}(\underline{p})}\right) & ,|\underline{p}| \neq 0 \\
\cos \left(p_{0}\right) & ,|\underline{p}|=0\end{cases}
\end{aligned}
$$

are called the quaternion sine and cosine functions.

Corollary 4.1. (see [6, 7]) The quaternion sine and cosine functions admit the following representations:

$$
\begin{aligned}
& \sin (p)=\sin \left(p_{0}\right) \cos (\underline{p})+\cos \left(p_{0}\right) \sin (\underline{p}), \\
& \cos (p)=\cos \left(p_{0}\right) \cos (\underline{p})-\sin \left(p_{0}\right) \sin (\underline{p}) .
\end{aligned}
$$

The usual identities obey, such as $\sin (p)=-\sin (-p)$ and $\cos (p)=\cos (-p)$. A straightforward computation shows that $\sin ^{2}(p)+\cos ^{2}(p)=1_{\mathbb{H}}$. However, one has to pay attention to the fact that the quaternion trigonometric functions do in general not satisfy the sum and difference formulae: Take for example $p=\mathfrak{i}+\mathfrak{j}$, and $\sin (\mathfrak{i}+\mathfrak{j})=\frac{(\mathfrak{i}+\mathfrak{j})}{\sqrt{2}} \sinh (\sqrt{2}) \neq(\mathfrak{i}+\mathfrak{j}) \sinh (1) \cosh (1)=\sin (\mathfrak{i}) \cos (\mathfrak{j})+$ $\cos (\mathfrak{i}) \sin (\mathfrak{j})$.

We may now have a look to the zeros of the quaternion sine and cosine functions. Since $\operatorname{sgn}(\overline{\bar{p}})=-\operatorname{sgn}(p)$, it can be easily shown that

$$
|\sin (p)|^{2}=\sin ^{2}\left(p_{0}\right)+\sinh ^{2}|\underline{p}|
$$

and

$$
|\cos (p)|^{2}=\cos ^{2}\left(p_{0}\right)+\cosh ^{2}|\underline{p}|-1 \text {. }
$$

Since $\sin ^{2}\left(p_{0}\right)$ (resp. $\left.\cos ^{2}\left(p_{0}\right)\right)$ and $\sinh ^{2}|\underline{p}|$ are both nonnegative real numbers, it is evident that the structural equations are satisfied if and only if $\sin \left(p_{0}\right)=0\left(\right.$ resp. $\left.\cos \left(p_{0}\right)=0\right)$ and $\sinh |p|=0$. As is well known, $\sin \left(p_{0}\right)=0$ when $p_{0}=n \pi, n=0, \pm 1, \pm 2, \ldots\left(\right.$ resp. $\cos \left(p_{0}\right)=0$ when $\left.p_{0}=\frac{(2 n+1) \pi}{2}, n=0, \pm 1, \pm 2, \ldots\right)$, and $\sinh |\underline{p}|=0$ only when $|\underline{p}|=0$. Therefore the quaternion trigonometric functions have only the zeros known for the real functions.

Now we turn our attention to inequalities involving the quaternion trigonometric functions.

Theorem 4.1. Let $\mathrm{p}$ be a real quaternion such that $|\underline{p}| \leq \ln (1+\sqrt{2})$. Then

$$
|\sin (p)| \leq \sqrt{p_{0}^{2}+1}
$$


Proof. Notice that $\sin ^{2}\left(p_{0}\right) \leq p_{0}^{2}$, and $\sinh ^{2}(|\underline{p}|) \leq 1$ for every $|\underline{p}| \in[0, \ln (1+\sqrt{2})]$. Based on the representation 4.1), it follows $|\sin (p)|^{2} \leq p_{0}^{2}+1$.

Theorem 4.2. Let $\mathrm{p}$ be a real quaternion such that $|\underline{p}| \geq \ln (1+\sqrt{2})$, and $\mathrm{p}_{0} \leq-\sqrt{6}$ or $\mathrm{p}_{0} \in[0, \sqrt{6}]$. Then

$$
|\sin (p)| \geq \sqrt{1+\left(p_{0}-\frac{p_{0}^{3}}{6}\right)^{2}}
$$

Proof. Note, in passing, that $\sin ^{2}\left(p_{0}\right) \geq\left(p_{0}-\frac{p_{0}^{3}}{6}\right)^{2}$ when $p_{0} \leq-\sqrt{6}$ or $p_{0} \in[0, \sqrt{6}]$, and $\sinh ^{2}(|\underline{p}|) \geq 1$ for $|\underline{p}| \leq \ln (1+\sqrt{2})$. In summary, we set

$$
|\sin (p)|^{2} \geq 1+\left(p_{0}-\frac{p_{0}^{3}}{6}\right)^{2} .
$$

Theorem 4.3. Let $\mathrm{p}$ be a real quaternion such that $\mathrm{p}_{0} \in[0, \sqrt{2}]$. Then

$$
|\cos (p)| \geq 1-\frac{p_{0}^{2}}{2}
$$

Proof. Let $p_{0} \in[0, \sqrt{2}]$, it is clear that $\cos ^{2}\left(p_{0}\right) \geq\left(1-\frac{p_{0}^{2}}{2}\right)^{2}$. Based on the relation 4.2 , we conclude that

$$
|\cos (p)|^{2} \geq\left(1-\frac{p_{0}^{2}}{2}\right)^{2}+1-1=\left(1-\frac{p_{0}^{2}}{2}\right)^{2}
$$

In closing this section, we note that the usual definitions of the other trigonometric functions are taken:

Definition 4.2. For $p \in \mathbb{H} \backslash\{(n+1 / 2) \pi: n=0, \pm 1, \pm 2, \ldots\}$, the functions $\tan (p)$ and $\sec (p)$ defined respectively by

$$
\tan (p)=\frac{\sin (p)}{\cos (p)}, \quad \text { and } \quad \sec (p)=\frac{1}{\cos (p)},
$$

are called the quaternion tangent and secant functions.

Definition 4.3. For $\mathrm{p} \in \mathbb{H} \backslash\{\mathrm{n} \pi: \mathrm{n}=0, \pm 1, \pm 2, \ldots\}$, the functions $\cot (\mathrm{p})$ and $\csc (\mathrm{p})$ defined respectively by

$$
\cot (p)=\frac{\cos (p)}{\sin (p)}, \quad \text { and } \quad \csc (p)=\frac{1}{\sin (p)},
$$

are called the quaternion cotangent and cosecant functions. 
Theorem 4.4. Let $\mathrm{p}$ be a real quaternion such that $|\underline{\mathrm{p}}| \leq \ln (1+\sqrt{2})$, and $\mathrm{p}_{\mathrm{o}} \in[0, \sqrt{2})$. Then

$$
|\tan (p)| \leq \frac{2 \sqrt{1+p_{0}^{2}}}{2-p_{0}^{2}} .
$$

Proof. The proof follows from Theorems 4.1 and 4.3 .

Theorem 4.5. Let $\mathrm{p}$ be a real quaternion such that $|\underline{\mathrm{p}}| \leq \ln (1+\sqrt{2})$, and $\mathrm{p}_{\mathrm{o}} \in[0, \sqrt{2}]$. Then

$$
|\cot (p)| \geq \frac{2-p_{0}^{2}}{2 \sqrt{p_{0}^{2}+1}} .
$$

Proof. It follows from Theorems 4.1 and 4.3 .

\section{Hyperbolic revisited}

The quaternion hyperbolic sine and cosine functions may be defined using the quaternion exponential function as follows:

Definition 5.1. The functions $\sinh (p)$ and $\cosh (p)$ defined respectively by

$$
\sinh (p)=\frac{e^{\mathfrak{p}}-e^{-\mathfrak{p}}}{2}, \quad \cosh (p)=\frac{e^{\mathfrak{p}}+e^{-\mathfrak{p}}}{2},
$$

are called the quaternion sine and cosine hyperbolic functions.

Similarly to their counterparts for real and complex input the quaternion hyperbolic sine and cosine are respectively, odd and even functions. A straightforward computation shows that $\cosh ^{2}(p)-\sinh ^{2}(p)=1_{\mathbb{H}}$. However, we must keep clearly in mind that the quaternion hyperbolic functions do in general not satisfy the sum and difference formulae, unless $p_{1}$ and $p_{2}$ commute. It can be settled by a simple example which we sketch briefly. Take for example $p=\mathfrak{i}-\mathfrak{j}$, then $\sinh (\mathfrak{i}-\mathfrak{j})=\frac{(\mathfrak{i}-\mathbf{j})}{\sqrt{2}} \sin (\sqrt{2}) \neq(\mathfrak{i}-\mathfrak{j}) \cos (1) \sin (1)=\sinh (\mathfrak{i}) \cosh (\mathfrak{j})-\cosh (\mathfrak{i}) \sinh (\mathfrak{j})$.

Remark 5.1. The zeros of the function $\sinh (\mathrm{p})$ are the solutions for $\mathrm{p}$ of the equation $\mathrm{e}^{\mathrm{p}}=\mathrm{e}^{-\mathrm{p}}$, which can be rewritten as $e^{2 p}=1_{\mathbb{H}}$. Since $e^{2 p}=1_{\mathbb{H}}$ only when $2 \underline{p}$ is an integer multiple of $2 \pi \operatorname{sgn}(\underline{p})$, we conclude that the zeros of $\sinh (p)$ are the numbers $n \pi \operatorname{sgn}(\underline{p}), n=0, \pm 1, \pm 2, \ldots$ Similarly, the zeros of $\cosh (\mathrm{p})$ are the solutions for $\mathrm{p}$ of the equation $\mathrm{e}^{\mathrm{p}}=-\mathrm{e}^{-\mathrm{p}}$, which can be rewritten as $e^{2 p}=-1_{\mathbb{H}}$, or as $e^{2 p-\operatorname{sgn}(\underline{p}) \pi}=1_{\mathbb{H}}$ (since $\left.e^{\operatorname{sgn}(\underline{p}) \pi}=-1_{\mathbb{H}}\right)$. It follows that the zeros of $\cosh (\mathrm{p})$ are the numbers $\left(\mathrm{n}+\frac{1}{2}\right) \pi \operatorname{sgn}(\underline{\mathrm{p}})$ with $\mathrm{n}=0, \pm 1, \pm 2, \ldots$

We proceed to study inequalities involving the quaternion sine and cosine hyperbolic functions. 
Theorem 5.1. Let $\mathrm{p}$ be a real quaternion. Then

$$
|\sinh (p)| \leq 2 \cosh \left(p_{0}\right), \quad \text { and } \quad|\cosh (p)| \leq 2 \cosh \left(p_{0}\right) .
$$

Proof. From Theorem 3.1 we set $\left|e^{\mathfrak{p}}\right| \leq 2 e^{p_{0}}$. In particular, it holds $\left|e^{-\mathfrak{p}}\right| \leq 2 e^{-p_{0}}$. Now, relying on Defintion 5.1 it follows $|\sinh (p)| \leq 2 \cosh \left(p_{0}\right)$. In a similar manner, we obtain the second inequality of our theorem.

Analogously to quaternion trigonometric functions, we next define the quaternion tangent, cotangent, secant, and cosecant hyperbolic functions using the quaternion hyperbolic sine and cosine.

Definition 5.2. For $\mathrm{p} \in \mathbb{H} \backslash\left\{\left(\mathrm{n}+\frac{1}{2}\right) \pi \operatorname{sgn}(\underline{\mathrm{p}}): \mathrm{n}=0, \pm 1, \pm 2, \ldots\right\}$, the functions $\tanh (\mathrm{p})$ and $\operatorname{sech}(p)$ defined respectively by

$$
\tanh (p)=\frac{\sinh (p)}{\cosh (p)}, \quad \text { and } \quad \operatorname{sech}(p)=\frac{1}{\cosh (p)}
$$

are called the quaternion tangent and secant hyperbolic functions.

Definition 5.3. For $\mathrm{p} \in \mathbb{H} \backslash\{\mathrm{n} \pi \operatorname{sgn}(\underline{\mathrm{p}}): \mathrm{n}=0, \pm 1, \pm 2, \ldots\}$, the functions $\operatorname{coth}(\mathrm{p})$ and $\operatorname{csch}(\mathrm{p})$ defined respectively by

$$
\operatorname{coth}(p)=\frac{\cosh (p)}{\sinh (p)}, \quad \text { and } \quad \operatorname{csch}(p)=\frac{1}{\sinh (p)},
$$

are called the quaternion cotangent and cosecant functions.

\section{Inverse hyperbolic and trigonometric functions revisited}

The main focus of this section is to study the inverses of the quaternion trigonometric and hyperbolic functions, and their properties. Since the quaternion trigonometric and hyperbolic functions are defined in terms of the quaternion exponential function $e^{p}$, their inverses are necessarily multiple-valued and may be computed via the quaternion natural logarithm function $\ln (p)$. We summarize this discussion in the following definition.

Definition 6.1. The multiple-valued functions $\sinh ^{-1}(p)$ and $\cosh ^{-1}(p)$ defined respectively by

$$
\sinh ^{-1}(p)=\ln \left(p+\sqrt{p^{2}+1}\right), \quad \cosh ^{-1}(p)=\ln \left(p+\sqrt{p^{2}-1}\right)
$$

are called the quaternion inverse hyperbolic sine and cosine.

These functions have two sources of multivaluedness; one due to the quaternion natural logarithm function $\ln (p)$, the other due to the involved quaternion power functions. It is evident that 
the quaternion inverse hyperbolic sine and cosine can be made single-valued by specifying a single value of the quaternion logarithm and a single value of the functions $\left(p^{2}+1\right)^{1 / 2}$ and $\left(p^{2}-1\right)^{1 / 2}$, respectively. We see at the same time that a branch of a quaternion inverse hyperbolic function may be obtained by choosing a branch of the quaternion logarithm and a branch of a quaternion power function. In spite of its local multiplevaluedness, the $\ln (p)$ function has an infinite number of branches, hence so do the quaternion inverse hyperbolic sine and cosine. In addition, the quaternion inverse hyperbolic functions have branch point-solutions to the equations $p^{2} \pm 1=0_{\mathbb{H}}$, because the functions $\left(p^{2}+1\right)^{1 / 2}$ and $\left(p^{2}-1\right)^{1 / 2}$ have no solutions of $\left(p^{2}+1\right)^{1 / 2}=0_{\mathbb{H}}$ and $\left(p^{2}-1\right)^{1 / 2}=0_{\mathbb{H}}$, respectively.

Theorem 6.1. Let $p$ be a real quaternion such that $|p|^{4}-|p|^{2}+2 p_{0}^{2} \geq 0$. The following inequalities are valid:

(1) $\left|\sinh ^{-1}(p)\right| \leq \sqrt{1+2\left(|p|^{4}-|p|^{2}+2 p_{0}^{2}\right)}+\pi$,

(2) $\left|\cosh ^{-1}(p)\right| \leq \sqrt{1+2\left(|p|^{4}-|p|^{2}+2 p_{0}^{2}\right)}+\pi$,

(3) $\left|\sinh ^{-1}(p)\right| \leq 1+2\left(|p|^{4}-|p|^{2}+2 p_{0}^{2}\right)-\frac{\left(\sqrt{1+2\left(|p|^{4}-|p|^{2}+2 p_{0}^{2}\right)}\right)^{2}}{2}$ $+\frac{\left(\sqrt{1+2\left(|p|^{4}-|p|^{2}+2 p_{0}^{2}\right)}\right)^{3}}{3}+\pi$

(4) $\left|\cosh ^{-1}(\mathrm{p})\right| \leq 1+2\left(|\mathrm{p}|^{4}-|\mathrm{p}|^{2}+2 \mathrm{p}_{0}^{2}\right)-\frac{\left(\sqrt{1+2\left(|\mathbf{p}|^{4}-|\mathbf{p}|^{2}+2 \mathrm{p}_{0}^{2}\right)}\right)^{2}}{2}$ $+\frac{\left(\sqrt{1+2\left(|p|^{4}-|p|^{2}+2 p_{0}^{2}\right)}\right)^{3}}{3}+\pi$

(5) $\left|\sinh ^{-1}(p)\right| \leq \sum_{k=1}^{2 n-1}(-1)^{k+1} \frac{\left.\left(\sqrt{1+2\left(|p|^{4}-|p|^{2}+2 p_{0}^{2}\right.}\right)\right)^{k}}{k}+\pi, \quad n \in \mathbb{N}$,

(6) $\left|\cosh ^{-1}(p)\right| \leq \sum_{k=1}^{2 n-1}(-1)^{k+1} \frac{\left(\sqrt{1+2\left(|p|^{4}-|p|^{2}+2 p_{0}^{2}\right)}\right)^{k}}{k}+\pi, \quad n \in \mathbb{N}$.

Proof. Let $\mathrm{p}:=\mathrm{p}_{0}+\mathrm{p}_{1} \mathbf{i}+\mathrm{p}_{2} \mathbf{j}+\mathrm{p}_{3} \mathbf{k}$ be a real quaternion. By Definition 3.4 we find the following representation for $p+\sqrt{p^{2}+1}$ :

$$
\begin{aligned}
& p+\sqrt{\left(p_{0}^{2}+1-|\underline{p}|^{2}\right)^{2}+4 p_{0}^{2}|\underline{p}|^{2}}\left(\cos \left|\frac{1}{2} \arccos \frac{p_{0}^{2}+1-|\underline{p}|^{2}}{\left(p_{0}^{2}+1-|\underline{p}|^{2}\right)^{2}+4 p_{0}^{2}|\underline{p}|^{2}}\right|\right. \\
+ & \left.\operatorname{sgn}(\underline{p}) \sin \left|\frac{1}{2} \arccos \frac{p_{0}^{2}+1-|\underline{p}|^{2}}{\left(p_{0}^{2}+1-|\underline{p}|^{2}\right)^{2}+4 p_{0}^{2}|\underline{p}|^{2}}\right|\right) .
\end{aligned}
$$


With $A$ denoting the term $\sqrt{\left(p_{0}^{2}+1-|\underline{p}|^{2}\right)^{2}+4 p_{0}^{2}|\underline{p}|^{2}}$, we have, on account of 6.1 :

$$
\begin{aligned}
& \left|p+\sqrt{p^{2}+1}\right|^{2}=\left(p_{0}+A \cos \left|\frac{1}{2} \arccos \frac{p_{0}^{2}+1-|\underline{p}|^{2}}{\left(p_{0}^{2}+1-|\underline{p}|^{2}\right)^{2}+4 p_{0}^{2}|\underline{p}|^{2}}\right|\right)^{2} \\
+ & \left(p_{1}+A \frac{p_{1}}{\sqrt{p_{1}^{2}+p_{2}^{2}+p_{3}^{2}}} \sin \left|\frac{1}{2} \arccos \frac{p_{0}^{2}+1-|\underline{p}|^{2}}{\left(p_{0}^{2}+1-|\underline{p}|^{2}\right)^{2}+4 p_{0}^{2}|\underline{p}|^{2}}\right|\right)^{2} \\
+ & \left(p_{2}+A \frac{p_{2}}{\sqrt{p_{1}^{2}+p_{2}^{2}+p_{3}^{2}}} \sin \left|\frac{1}{2} \arccos \frac{p_{0}^{2}+1-|\underline{p}|^{2}}{\left(p_{0}^{2}+1-|\underline{p}|^{2}\right)^{2}+4 p_{0}^{2}|\underline{p}|^{2}}\right|\right)^{2} \\
+ & \left(p_{3}+A \frac{p_{3}}{\sqrt{p_{1}^{2}+p_{2}^{2}+p_{3}^{2}}} \sin \left|\frac{1}{2} \arccos \frac{p_{0}^{2}+1-|\underline{p}|^{2}}{\left(p_{0}^{2}+1-|\underline{p}|^{2}\right)^{2}+4 p_{0}^{2}|\underline{p}|^{2}}\right|\right)^{2}
\end{aligned}
$$

Now we make use of the standard inequality: $(a+b)^{2} \leq 2\left(a^{2}+b^{2}\right)$. Hence we obtain

$$
\begin{aligned}
& \left|p+\sqrt{p^{2}+1}\right|^{2} \leq 2\left[p_{0}^{2}+\left(A \cos \left|\frac{1}{2} \arccos \frac{p_{0}^{2}+1-|\underline{p}|^{2}}{\left(p_{0}^{2}+1-|\underline{p}|^{2}\right)^{2}+4 p_{0}^{2}|\underline{p}|^{2}}\right|\right)^{2}\right. \\
+ & p_{1}^{2}+\left(A \frac{p_{1}}{\sqrt{p_{1}^{2}+p_{2}^{2}+p_{3}^{2}}} \sin \left|\frac{1}{2} \arccos \frac{p_{0}^{2}+1-|\underline{p}|^{2}}{\left(p_{0}^{2}+1-|\underline{p}|^{2}\right)^{2}+4 p_{0}^{2}|\underline{p}|^{2}}\right|\right)^{2} \\
+ & p_{2}^{2}+\left(A \frac{p_{2}}{\sqrt{p_{1}^{2}+p_{2}^{2}+p_{3}^{2}}} \sin \left|\frac{1}{2} \arccos \frac{p_{0}^{2}+1-|\underline{p}|^{2}}{\left(p_{0}^{2}+1-|\underline{p}|^{2}\right)^{2}+4 p_{0}^{2}|\underline{p}|^{2}}\right|\right)^{2} \\
+ & \left.p_{3}^{2}+\left(A \frac{p_{3}}{\sqrt{p_{1}^{2}+p_{2}^{2}+p_{3}^{2}}} \sin \left|\frac{1}{2} \arccos \frac{p_{0}^{2}+1-|\underline{p}|^{2}}{\left(p_{0}^{2}+1-|\underline{p}|^{2}\right)^{2}+4 p_{0}^{2}|\underline{p}|^{2}}\right|\right)^{2}\right] \\
= & 2\left(|p|^{4}-|p|^{2}+2 p_{0}^{2}+1\right),
\end{aligned}
$$

that is,

$$
\left|p+\sqrt{p^{2}+1}\right|^{2} \leq 1+1+2\left(|p|^{4}-|p|^{2}+2 p_{0}^{2}\right) .
$$

Furthermore, using the inequality $\sqrt{a+b} \leq \sqrt{a}+\sqrt{b}$ for $a \geq 0$ and $b \geq 0$, the following further inequality is now immediate:

$$
\log _{e}\left|p+\sqrt{p^{2}+1}\right| \leq \log _{e}\left(1+\sqrt{1+2\left(|p|^{4}-|p|^{2}+2 p_{0}^{2}\right)}\right) .
$$

We may now use the inequality $\ln (1+x) \leq x$ for $x \geq 0$ to obtain:

$$
\log _{e}\left|p+\sqrt{p^{2}+1}\right| \leq \sqrt{1+2\left(|p|^{4}-|p|^{2}+2 p_{0}^{2}\right)}
$$


from which we find,

$$
\left|\operatorname{Ln}\left(p+\sqrt{p^{2}+1}\right)\right| \leq \sqrt{1+2\left(|p|^{4}-|p|^{2}+2 p_{0}^{2}\right)}+\pi .
$$

We have thus obtain the following further inequality:

$$
\left|\sinh ^{-1}(p)\right| \leq \sqrt{1+2\left(|p|^{4}-|p|^{2}+2 p_{0}^{2}\right)}+\pi
$$

In a similar way we obtain the inequality for $\cosh ^{-1}(p)$. The proofs of the remaining statements follow from the aforementioned inequalities used in the proof of Theorem 3.3 .

The quaternion inverse hyperbolic tangent can be now introduced.

Definition 6.2. The multiple-valued function $\tanh ^{-1}(\mathrm{p})$ defined respectively by

$$
\tanh ^{-1}(p)=\frac{\ln (1+p)-\ln (1-p)}{2},
$$

is called the quaternion inverse hyperbolic tangent.

All this being established, we can now introduce the inverse trigonometric sine, cosine and tangent quaternion functions.

Definition 6.3. The multiple-valued functions $\sin ^{-1}(p)$ and $\cos ^{-1}(p)$ defined respectively by

$$
\begin{aligned}
& \sin ^{-1}(p)=\operatorname{sgn}(\underline{p}) \sinh ^{-1}(p \operatorname{sgn}(\underline{p})) \\
& \cos ^{-1}(p)=\operatorname{sgn}(\underline{p}) \cosh ^{-1}(p)
\end{aligned}
$$

are called the quaternion inverse sine and cosine.

Definition 6.4. The multiple-valued function $\tan ^{-1}(\mathrm{p})$ defined respectively by

$$
\tan ^{-1}(p)=\operatorname{sgn}(\underline{p}) \tanh ^{-1}(p \operatorname{sgn}(\underline{p}))
$$

is called the quaternion inverse tangent.

\subsection{Acknowledgements}

The first author's research is supported by the Ministry of Science and Education of Bulgaria, Grant DD-VU 02/90. The second author acknowledges financial support from Foundation for Science and Technology (FCT) via the post-doctoral grant SFRH/BPD/66342/2009.

Received: May 2011. Revised: June 2011. 


\section{References}

[1] R. Fueter. Analytische Funktionen einer Quaternionenvariablen. Comment. Math. Helv., 4, 9-20 (1932).

[2] R. Fueter: Die Funktionentheorie der Differentialgleichungen $\Delta \mathrm{u}=0$ und $\Delta \Delta \mathrm{u}=0$ mit vier reellen Variablen. Comm. Math. Helv. 7: 307-330 (1935).

[3] R. Fueter. Über die analytische Darstellung der regulären Funktionen einer Quaternionenvariablen. Comment. Math. Helv., 8, 371-378 (1935).

[4] R. Fueter. Functions of a Hyper Complex Variable. Lecture notes written and supplemented by E. Bareiss, Math. Inst. Univ. Zürich, Fall Semester, 1949.

[5] K. Gürlebeck and W. Sprössig. Quaternionic Analysis and Elliptic Boundary Value Problems. Akademie Verlag, Berlin, 1989.

[6] K. Gürlebeck and W. Sprössig. Quaternionic Calculus for Physicists and Engineers. John Wiley and Sons, Chichester, 1997.

[7] K. Gürlebeck, K. Habetha and W. Sprößig. Holomorphic Functions in the Plane and ndimensional Space, Birkhäuser Verlag, Basel - Boston - Berlin, 2008.

[8] V. Kravchenko. Applied quaternionic analysis. Research and Exposition in Mathematics. Lemgo: Heldermann Verlag, Vol. 28, 2003.

[9] H. Leutwiler, Quaternionic analysis in $\mathbb{R}^{3}$ versus its hyperbolic modification, Brackx, F., Chisholm, J.S.R. and Soucek, V. (ed.). NATO Science Series II. Mathematics, Physics and Chemistry, vol. 25, Kluwer Academic Publishers, Dordrecht, Boston, London, 2001, pp. 193211.

[10] H. Malonek. Quaternions in applied sciences: A historical perspective of a mathematical concept. In Proceedings of the International Conference on the Applications of Computer Science and Mathematics in Architecture and Civil Engineering. Bauhaus-Universität Weimar (2003).

[11] M. Shapiro and N. L. Vasilevski. Quaternionic $\psi$-hyperholomorphic functions, singular operators and boundary value problems I. Complex Variables, Theory Appl., 1995.

[12] M. Shapiro and N. L. Vasilevski. Quaternionic $\psi$-hyperholomorphic functions, singular operators and boundary value problems II. Complex Variables, Theory Appl., 1995.

[13] W. Sprössig. On Operators and Elementary Functions in Clifford Analysis. Zeitschrift fr Analysis und ihre Anwendungen, Vol. 18, No. 2, pp. 349-360 (1999).

[14] A. Sudbery. Quaternionic analysis. Math. Proc. Cambridge Phil. Soc. 85: pp. 199-225 (1979). 